\title{
A quadratically convergent algorithm for finding the largest eigenvalue of a nonnegative homogeneous polynomial map
}

\author{
Qin Ni · Liqun Qi
}

Received: 11 June 2013 / Accepted: 2 June 2014 / Published online: 10 June 2014

C Springer Science+Business Media New York 2014

\begin{abstract}
In this paper we propose a quadratically convergent algorithm for finding the largest eigenvalue of a nonnegative homogeneous polynomial map where the Newton method is used to solve an equivalent system of nonlinear equations. The semi-symmetric tensor is introduced to reveal the relation between homogeneous polynomial map and its associated semi-symmetric tensor. Based on this relation a globally and quadratically convergent algorithm is established where the line search is inserted. Some numerical results of this method are reported.
\end{abstract}

Keywords Nonnegative homogenous polynomial mapping - Nonnegative tensors · Eigenvalue of polynomial mapping $\cdot$ Newton method

\section{Introduction}

Eigenvalues for higher order tensors were introduced in [7,9,13]. Meanwhile, the PerronFrobenius theorem for nonnegative tensors is related to measuring higher order connectivity in hypergraphs $[1,2,6]$. The Perron-Frobenius theorem is further extended to multilinear forms and polynomial map with nonnegative coefficients [5,17]. Applications of eigenvalues

Qin Ni: His work was supported by National Science Foundation of China (11071117) and Jiangsu Province (SBK2014020180).

Liqun Qi: His work was supported by the Hong Kong Research Grant Council (Grant No. PolyU 501909, 502510, 502111 and 501212).

Q. Ni (凶)

Department of Mathematics, Nanjing University of Aeronautics and Astronautics, Nanjing 210016,

People's Republic of China

e-mail: niqfs@nuaa.edu.cn

L. Qi

Department of Applied Mathematics, The Hong Kong Polytechnic University, Kowloon, Hong Kong e-mail: liqun.qi@polyu.edu.hk 
of tensors and multivariate polynomial include signal processing [14], medical resonance imaging [15,16], higher-order Markov chains [10], positive definiteness of even-order multivariate forms in automatic control [11]. Subsequently, an algorithm for finding the largest eigenvalue of a nonnegative tensor, extending the Collatz's method for nonnegative matrices, was proposed by [10], its variations and linear convergence were studied in $[8,18,19]$.

In this paper we consider the polynomial map $P=\left(p_{1}, p_{2}, \cdots, p_{n}\right)^{T}: \Re^{n} \rightarrow \Re^{n}$ where each $p_{i}$ is a homogeneous polynomial of degree $d \geq 1$ with nonnegative coefficients. For convenience, $P$ is called a nonnegative homogeneous polynomial map of degree $d$.

Definition 1.1 Assume that $P$ is a nonnegative homogeneous polynomial map of degree $d$. We say that $(\lambda, x) \in R \times\left(R^{n} \backslash\{0\}\right)$ is an eigenvalue-eigenvector pair of $P$ if

$$
P(x)=\lambda x^{[d]} .
$$

Here, $x^{[p]}=\left[x_{1}^{p}, x_{2}^{p}, \cdots, x_{n}^{p}\right]^{T}$.

This definition is from [5] where a minor change is made. If we let $F(x)=P(x)^{[1 / d]}$, then $(\lambda, x) \in R \times\left(R^{n} \backslash\{0\}\right)$ is an eigenvalue-eigenvector pair of $P$ if and only if $\left(\lambda^{[1 / d]}, x\right) \in$ $R \times\left(R^{n} \backslash\{0\}\right)$ is an eigenvalue-eigenvector pair of $F$.

If $P$ is a homogeneous polynomial map of degree $d$, then there exists a $(d+1)$-order $n$-dimensional tensor $\mathcal{A}$ defined by

$$
\mathcal{A}=\left(a_{i_{0} i_{1} \cdots i_{d}}\right), a_{i_{0} i_{1} \cdots i_{d}} \in \mathfrak{R}, 1 \leq i_{0}, i_{1}, \cdots, i_{d} \leq n,
$$

such that $P(x)=\mathcal{A} x^{d}$, where

$$
\mathcal{A} x^{d}=\left(\sum_{i_{1} i_{2}, \cdots, i_{d}=1}^{n} a_{i_{0} i_{1} i_{2} \cdots i_{d}} x_{i_{1}} x_{i_{2}} \cdots x_{i_{d}}\right)_{1 \leq i_{0} \leq n} .
$$

We call $\mathcal{A}$ an associated tensor of $P$.

In addition, $\mathcal{A}$ is called nonnegative (or, respectively, positive) if $a_{i_{0} i_{1} \cdots i_{d}} \geq 0$ (or, respectively, $\left.a_{i_{0} i_{1} \cdots i_{d}}>0\right)$ ). A nonnegative tensor $\mathcal{A}$ is called reducible if there exists a nonempty proper index subset $I \subset\{1,2, \cdots, n\}$ such that

$$
\mathcal{A}_{i_{0} i_{1} \cdots i_{d}}=0, \forall i_{0} \in I, \forall i_{1}, \cdots i_{d} \notin I .
$$

If $\mathcal{A}$ is not reducible, then we call $\mathcal{A}$ irreducible. It is clear that if $\mathcal{A}$ is nonnegative, then $P=\mathcal{A} x^{d}$ is a nonnegative homogeneous polynomial map of degree $d$.

In [5], the Perron-Frobenius theorem is extended to a nonnegative homogeneous polynomial map.

Theorem 1.1 ([5]) Let $P$ and $\mathcal{A}$ be a nonnegative homogeneous polynomial map of degree $d$ and its associated tensor, respectively. Assume that $\mathcal{A}$ is irreducible. Then we have

(1) there are an unique scalar $\lambda$ and a positive vector $x$ which satisfy (1.1) and

$$
\lambda=\inf _{y \in \Re_{++}^{n}} \max _{1 \leq i \leq n} \frac{P_{i}(y)}{y_{i}^{d}}=\sup _{y \in \Re_{+}^{n} \backslash\{0\}} \min _{1 \leq i \leq n, y_{i} \neq 0} \frac{P_{i}(y)}{y_{i}^{d}},
$$

where $P_{i}(y)$ is the $i$-th component of $P(x)$.

(2) If $\omega \in C$ and $v=\left(v_{1}, \cdots, v_{n}\right)^{T} \in C^{n} \backslash\{0\}$ are such that $P(v)=\omega v^{[d]}$, then $|\omega| \leq \lambda$.

This theorem is a combination of Corollaries 4.2 and 4.3 in [5]. In [5], the results applied to a more general condition, called the weak irreducibility condition. Here we only state them 
under the irreducibility condition. The definitions of irreducibility and weak irreducibility of a polynomial map may refer to [5].

In [5], an algorithm for finding the largest eigenvalue of a nonnegative polynomial map was also proposed by Friedland, Gauber and Han. We call it the FGH method for short. Local linear convergence of the FGH method was established in [5]. In this paper we want to study the relation between a nonnegative homogenous polynomial $\mathcal{A} x^{d}$ and its corresponding tensor $\mathcal{A}$, and propose a quadratically convergent algorithm for finding the largest eigenvalue of that nonnegative homogeneous polynomial map. The semi-symmetric tensor is introduced to reveal the relation between a homogeneous polynomial map and its associated tensor, while the property of semi-symmetric tensor gives a sound foundation for a new method for computing the eigenvalues of a homogenous polynomial map or a tensor.

This paper is organized as follows. We discuss the relation between a homogenous polynomial map and its associate semi-symmetric tensor in Sect.2. A Newton algorithm for computing the largest eigenvalue of a nonnegative homogenous polynomial map is proposed in Sect. 3. We establish quadratical and global convergence of the algorithm in Sect.4. In Sect. 5, we give some preliminary numerical test results.

Let $\mathfrak{R}_{+}^{n}=\left\{x \in \mathfrak{R}^{n} \mid x \geq 0\right\}, \mathfrak{R}_{++}^{n}=\left\{x \in \mathfrak{R}^{n} \mid x>0\right\}$. Denote $x \circ y$ as the product of $x$ and $y$ by $x \circ y=\left(x_{1} y_{1}, \cdots, x_{n} y_{n}\right)^{T}$ where $x, y \in \mathfrak{R}^{n}$. The tensor $\mathcal{A}$ is called symmetric if its entries are invariant under any permutation of their indices, i.e.

$$
a_{j_{0} j_{1} \cdots j_{d}}=a_{i_{0} i_{1} \cdots i_{d}},
$$

$j_{0} j_{1} \cdots j_{d}$ is any permutation of $i_{0} i_{1} \cdots i_{d}, 1 \leq i_{0}, i_{1}, \cdots, i_{d} \leq n$. A nonnegative tensor $\mathcal{A}$ is called primitive [3] if there exists a positive integer $k$ such that $F^{(k)}(x) \in \mathfrak{R}_{++}^{n}$ for any nonzero $x \in \Re_{+}^{n}$ where $F^{(1)}(x) \equiv F(x) \equiv\left(\mathcal{A} x^{d}\right)^{[1 / d]}, F^{(k)}(x)=F\left(F^{(k-1)}(x)\right)$ for $k=2,3, \cdots$.

\section{A nonnegative homogeneous polynomial map and its associated tensor}

In this section, we discuss the relation between a nonnegative homogeneous polynomial map and its associated tensor.

Example 2.1 Let $P(x)=\mathcal{A} x^{3}$, where $\mathcal{A}=\left(a_{i j k l}\right)$ is defined by

$$
\begin{aligned}
& a_{1111}=a_{2222}=2, a_{3333}=3 ; \\
& a_{1112}=1, a_{1121}=-2, a_{1211}=1 ; \\
& a_{1113}=2, a_{1131}=-1, a_{1311}=0 ; \\
& a_{2123}=-3, a_{2132}=2, a_{2321}=5 ; \\
& a_{i j k l}=0 \text { elsewhere. }
\end{aligned}
$$

Then

$$
P(x)=\left(\begin{array}{c}
2 x_{1}^{3}+x_{1}^{2} x_{3} \\
2 x_{2}^{3}+4 x_{1} x_{2} x_{3} \\
3 x_{3}^{3}
\end{array}\right)
$$

The above example shows that $P$ is nonnegative although its associated tensor $\mathcal{A}$ is not nonnegative.

In order to reveal the relation of a nonnegative homogeneous polynomial map and its associated tensor, a semi-symmetric tensor is introduced in the following. 
Definition 2.1 Assume that $d \geq 1$. An $(d+1)$-order $n$-dimensional tensor $\mathcal{A}$ is called semisymmetric if its entries are invariant under any permutation of their backward $d$ indices, i.e.

$$
a_{i_{0} j_{1} j_{2} \cdots j_{d}}=a_{i_{0} i_{1} \cdots i_{d}},
$$

$1 \leq i_{0} \leq n, j_{1} \cdots j_{d}$ is any permutation of $i_{1} \cdots i_{d}, 1 \leq i_{1}, \cdots, i_{d} \leq n$.

From the definition, it is easy to see that a semi-symmetric tensor is the same as the symmetric tensor except the first subscript, a symmetric tensor is semi-symmetric, matrix is always semi-symmetric.

In order to describe semi-symmetric tensors, we denote $\Omega_{k, d}$ by

$$
\Omega_{k, d}=\left\{\left(i_{k}, i_{k+1}, \cdots i_{d}\right): i_{k} \leq i_{k+1} \leq \cdots \leq i_{d}, i_{k}, i_{k+1}, \cdots i_{d}=1,2, \cdots, n\right\}
$$

and define the set of all permutations of $\left(i_{k} \cdots i_{d}\right)$ by

$$
C_{i_{k} \cdots i_{d}}=\left\{j_{k} j_{k+1} \cdots j_{d} \mid:\left(j_{k} j_{k+1} \cdots j_{d}\right) \text { is a permutations of }\left(i_{k} \cdots i_{d}\right)\right\},
$$

for $\left(i_{k} \cdots i_{d}\right) \in \Omega_{k, d}$. For a semi-symmetric tensor, only entries with subscripts $i_{0} i_{1} \cdots i_{d}$ for $i_{0}=1,2, \cdots, n$ and all $\left(i_{1} \cdots i_{d}\right) \in \Omega_{1, d}$ need to be given, other entries can be determined by (2.1).

Lemma 2.1 Let $P$ be a homogeneous polynomial map of degree $d$. Then there is an unique $(d+1)$-order $n$-dimensional semi-symmetric tensor $\mathcal{A}_{s}$ such that

$$
P(x)=\mathcal{A}_{s} x^{d} .
$$

Proof Each component in $P$ is a homogeneous polynomial of $d$ degree which includes

$$
u_{d}=\left(\begin{array}{c}
d+n-1 \\
n-1
\end{array}\right)
$$

different monomials, all these monomials of degree $d$ are ordered in a $u_{d}$-dimensional vector by the graded lexicographic order in the following

$$
r(x)=\left(x_{1}^{d}, x_{1}^{(d-1)} x_{2}, \cdots, x_{1}^{(d-1)} x_{n}, \cdots, x_{n}^{d}\right)^{T} .
$$

It is noted that $u_{d}$ is also the cardinal number of $\Omega_{1 d}$ defined in (2.2). Let the coefficients of all monomials in $P_{i}$ be $m_{i j}, j=1,2, \cdots, u_{d}, i=1, \cdots, n$. Then

$$
P(x)=\operatorname{Mr}(x),
$$

where $M=\left(m_{i j}\right) \in R^{n \times u_{d}}$. Now we define $(d+1)$-order $n$-dimensional semi-symmetric tensor $\mathcal{A}_{s}$ where

$$
a_{i i_{1} \cdots i_{d}}=\frac{m_{i j}}{\left|C_{i_{1} \cdots i_{d}}\right|},
$$

$i_{1} \cdots i_{d} \in \Omega_{1, d},\left|C_{i_{1} \cdots i_{d}}\right|$ is the cardinal number of $C_{i_{1} \cdots i_{d}}$ defined in (2.3), $j$ corresponds with the grlex order of $i_{1} \cdots i_{d} \in \Omega_{1, d}, j=1,2, \cdots, u_{d}$. Other entries of $\mathcal{A}_{s}$ are determined by (2.1). Hence we have 


$$
\begin{aligned}
\left(\mathcal{A}_{s} x^{d}\right)_{i} & =\sum_{j_{1}=1}^{n} \sum_{j_{2}=1}^{n} \cdots \sum_{j_{d}=1}^{n} a_{i j_{1} \cdots j_{d}} x_{j_{1}} \cdots x_{j_{d}} \\
& =\sum_{i_{1}=1}^{n} \sum_{i_{2}=i_{1}}^{n} \cdots \sum_{i_{d}=i_{d-1}}^{n}\left|C_{i_{1} \cdots i_{d}}\right| a_{i i_{1} \cdots i_{d}} x_{i_{1}} \cdots x_{i_{d}} \\
& =\sum_{\left(i_{1} i_{2} \cdots i_{d}\right) \in \Omega_{1 d}}\left|C_{i_{1} \cdots i_{d}}\right| a_{i i_{1} \cdots i_{d}} x_{i_{1}} \cdots x_{i_{d}} \\
& =e_{i}^{T} \operatorname{Mr}(x)=P_{i}(x),
\end{aligned}
$$

for $i=1, \cdots, n$. In (2.9), the second equality is obtained by combing same monomials with the same coefficient, and the fourth equality is from (2.6) and (2.8).

Now we prove the uniqueness. Let $\tilde{\mathcal{A}}$ be semi-symmetric such that $\tilde{\mathcal{A}} x^{d}=P(x)$. Since $\tilde{\mathcal{A}}$ is semi-symmetric, for $i_{1} \cdots i_{d} \in \Omega_{1, d}, j$ is the grlex order of $i_{1} \cdots i_{d} \in \Omega_{1, d}, \tilde{a}_{i i_{1} \cdots i_{d}}$ is also determined by (2.8). Hence $\tilde{\mathcal{A}}=\mathcal{A}_{s}$.

Afterward we call $\mathcal{A}_{s}$ in (2.4) the associated semi-symmetric tensor. From the definition of a nonnegative polynomial map and (2.7), we know that $P$ is nonnegative if and only if the coefficients of $P(x), m_{i j} \geq 0, i=1, \cdots, n ; j=1, \cdots, u_{d}$ which implies by (2.8) that $\mathcal{A}_{s} \geq 0$. Hence $P$ is nonnegative if and only if its associated semi-symmetric tensor $\mathcal{A}_{s}$ is nonnegative.

Example 2.1 (continued) Let $\mathcal{A}_{s}$ be the associated semi-symmetric tensor of $P$. Then

$$
\begin{gathered}
\tilde{a}_{1111}=\tilde{a}_{2222}=2, \tilde{a}_{3333}=3 ; \\
\tilde{a}_{1113}=\tilde{a}_{1131}=\tilde{a}_{1311}=\frac{1}{\left|C_{113}\right|}=\frac{1}{3} ; \\
\tilde{a}_{2123}=\tilde{a}_{2132}=\tilde{a}_{2213}=\tilde{a}_{2231}=\tilde{a}_{2312}=\tilde{a}_{2321}=\frac{4}{\left|C_{113}\right|}=\frac{4}{6}=\frac{2}{3} ; \\
\tilde{a}_{i j k l}=0 \text { elsewhere. }
\end{gathered}
$$

It is noted that $\mathcal{A}$ is neither nonnegative nor semi-symmetric, while $P$ and its associated semi-symmetric tensor $\mathcal{A}_{s}$ are nonnegative.

Hence, by means of nonnegative homogenous polynomial map we can extend nonnegative tensor in the following.

Definition 2.2 An $(d+1)$-order $n$-dimensional tensor $\mathcal{A}$ is called semi-nonnegative, if $P$ is nonnegative where $P(x)=\mathcal{A} x^{d}$.

The following is a corollary of Theorem 1.1.

Corollary 2.1 Assume that $(d+1)$-order $n$-dimensional tensor $\mathcal{A}$ is semi-nonnegative, and that $P$ is weakly irreducible where $P(x)=\mathcal{A} x^{d}$. Then the pair $\left(x^{*}, \lambda^{*}\right)$ in Eq. (1.1) satisfy (1) and (2) in Theorem 1.1.

In the next section, we propose an algorithm for computing the largest eigenvalue of nonnegative homogenous polynomial map.

\section{Algorithm for finding the largest eigenvalue of a nonnegative homogenous polynomial map}

In this section, we propose an algorithm for computing the largest eigenvalue of a nonnegative homogenous polynomial map. 
Let $\mathcal{U}$ be the $(d+1)$-order $n$-dimensional unit tensor whose entries are

$$
u_{i_{1} i_{2} \cdots i_{d}}= \begin{cases}1, & \text { if } i_{1}=i_{2}=\cdots=i_{d} \\ 0, & \text { otherwise. }\end{cases}
$$

Let $P$ be a nonnegative homogeneous polynomial map of degree $d, \mathcal{A}$ be the associated semi-symmetric tensor of $P$. Then

$$
P(x)+t x^{[d]}=(\mathcal{A}+t \mathcal{U}) x^{d},
$$

where $t>0$.

Lemma 3.1 Let $P$ be a nonnegative homogeneous polynomial map of degree $d, \mathcal{A}$ be the associated semi-symmetric tensor, $t>0$, and $P(x)+t x^{[d]}=(\mathcal{A}+t \mathcal{U}) x^{[d]}$.

(1) If $\mathcal{A}$ is irreducible, then $(\mathcal{A}+t \mathcal{U})$ is primitive.

(2) If $\lambda$ is the largest eigenvalue of $(\mathcal{A}+t \mathcal{U})$, then $\lambda-t$ is the largest eigenvalue of $P$.

Proof This lemma follows from Corollary 3 in [13] and Theorem 2.4 in [8].

We choose $\sigma>0$, define $F: R_{++}^{n+1} \mapsto R^{n+1}$

$$
F(x, \lambda)=\left(\begin{array}{c}
P(x)+\sigma x^{[d]}-\lambda x^{[d]} \\
\frac{1}{d+1}\left(1-\sum_{i=1}^{n} x_{i}^{d+1}\right)
\end{array}\right),
$$

where $P$ is nonnegative homogeneous of degree $\mathrm{d}$, and get an important relation between the solution of $F(x, \lambda)$ and the largest eigenvalue of $P$.

Lemma 3.2 Let $P$ and $\mathcal{A}$ be nonnegative homogeneous of degree $d$ and its associated semisymmetric tensor, respectively. Assume that $\mathcal{A}$ is irreducible. Then there is a pair $\left(\lambda^{*}, x^{*}\right)>0$ where $\lambda^{*}$ is the largest eigenvalue of $P$ and $\left(\lambda^{*}, x^{*}\right)$ satisfies (1.1), and thus there are $\tilde{\lambda}^{*}>0$ and $\tilde{x}^{*}>0$ such that $F\left(\tilde{x}^{*}, \tilde{\lambda}^{*}\right)=0$.

On the contrary, if there are $\tilde{\lambda}>0$ and $\tilde{x}>0$ such that $F(\tilde{x}, \tilde{\lambda})=0$, then $(\tilde{\lambda}-\sigma)$ is the largest eigenvalue of $P$.

Proof From Theorem 1.1, it follows that there exists a pair $\left(\lambda^{*}, x^{*}\right)>0$ where $\lambda^{*}$ is the largest eigenvalue of $P$ and $\left(\lambda^{*}, x^{*}\right)$ satisfies (1.1). Let

$$
c=\sum_{i=1}^{n}\left(x_{i}^{*}\right)^{d+1}, \tilde{x}=\frac{x^{*}}{\sqrt[d+1]{c}} .
$$

Then from (3.3) and direct computation we have that $\left(\tilde{x}, \lambda^{*}+\sigma\right)$ satisfies $F\left(\tilde{x}, \lambda^{*}+\sigma\right)=0$.

Assume that there exist $\tilde{\lambda}>0$ and $\tilde{x}>0$ such that $F(\tilde{x}, \tilde{\lambda})=0$. Then from the first equation of (3.3), we have

$$
\mathcal{A} \tilde{x}^{d}=(\tilde{\lambda}-\sigma) \tilde{x}^{[d]},
$$

i.e. $(\tilde{\lambda}-\sigma, \tilde{x})$ satisfies $(1.1)$. Because $\mathcal{A}$ is the associated semi-symmetric tensor of $P$, by Lemma 3.1

$$
P(\tilde{x})=\mathcal{A} \tilde{x}^{d}
$$

Combining with (3.4) we have

$$
P(\tilde{x})=(\tilde{\lambda}-\sigma) \tilde{x}^{[d]} .
$$

According to the assumption, $\mathcal{A}$ is irreducible. From Lemma 2.2 in [10], it follows that $\tilde{\lambda}-\sigma>0$ which implies that $\tilde{\lambda}-\sigma$ is the largest eigenvalue of $P$ from Theorem 1.1. 
Based on Lemma 3.2, the problem for finding the largest eigenvalue of $P$ is equivalent to solving (3.3). In the computation, $P(x)$ is replaced by $\mathcal{A}_{s} x^{d}$ where $\mathcal{A}_{s}$ is the associated semi-symmetric tensor of $P$. The computation of the Jacobian of of $F(x, \lambda)$ is determined in the following lemma.

Lemma 3.3 The Jacobian of $F(x, \lambda)$ is

$$
F^{\prime}(x, \lambda)=\left(\begin{array}{cc}
d\left(\left(\sigma \mathcal{U}+\mathcal{A}_{s}\right) x^{d-1}-\lambda \operatorname{diag}\left(x^{[d-1]}\right)\right) & -x^{[d]} \\
-\left(x^{[d]}\right)^{T} & 0
\end{array}\right),
$$

where

$$
\left(\mathcal{A}_{s} x^{(d-1)}\right)_{i j}=\sum_{i_{2}, \cdots, i_{d}=1}^{n} a_{i j i_{2} \cdots i_{d}} x_{i_{2}} \cdots x_{i_{d}}, i, j=1, \cdots, n .
$$

Proof From direct computation, we have

$$
\begin{aligned}
\left(\mathcal{A} x^{d}\right)_{i} & =\sum_{i_{1}, i_{2}, \cdots, i_{d}=1}^{n} a_{i i_{1} i_{2} \cdots i_{d}} x_{i_{1}} \cdots x_{i_{d}} \\
& =\sum_{i_{1} i_{2} \cdots i_{d} \in \Omega_{1, d}}\left|c_{i_{1} i_{2} \cdots i_{d}}\right| a_{i i_{1} i_{2} \cdots i_{d}} x_{i_{1}} \cdots x_{i_{d}} \\
& =\sum_{k=0}^{d} \sum_{i_{1} i_{2} \cdots i_{d} \in \Omega_{1, d}^{j, k}}\left|c_{i_{1} i_{2} \cdots i_{d}}\right| a_{i i_{1} i_{2} \cdots i_{d}} x_{i_{1}} \cdots x_{i_{d}},
\end{aligned}
$$

where $\Omega_{1, d}^{j, k}$ is a subset of $\Omega_{1, d}$, and its entries include $k j$ 's, $k=0,1, \cdots, d ; j=1, \cdots, n$. In (3.6), the second equality is obtained by the same deduction as (2.9). We compute the partial derivative and obtain that

$$
\begin{aligned}
\frac{\partial\left(\mathcal{A} x^{d}\right)_{i}}{\partial x_{j}} & =\sum_{k=1}^{d} \sum_{i_{1} i_{2} \cdots i_{d} \in \Omega_{1, d}^{j, k}}\left|c_{i_{1} i_{2} \cdots i_{d}}\right| a_{i i_{1} i_{2} \cdots i_{d}} \frac{\partial\left(x_{i_{1}} \cdots x_{i_{d}}\right)}{\partial x_{j}} \\
& =\sum_{k=0}^{d-1} \sum_{i_{2} \cdots i_{d} \in \Omega_{2, d}^{j, k}}\left|c_{i_{2} \cdots i_{d}}^{j}\right| a_{i j i_{2} \cdots i_{d}}(k+1)\left(x_{i_{2}} \cdots x_{i_{d}}\right),
\end{aligned}
$$

where $\left|c_{i_{2} \cdots i_{d}}^{j}\right|=\left|c_{j i_{2} \cdots i_{d}}\right|$, and the position of the first subscript $j$ may be changed so that the permutation of subscripts satisfies the order in $\Omega_{1, d}$. Let $i_{2} \cdots i_{d} \in \Omega_{2, d}^{j, k}, \beta_{j, k}$ be the number of permutation $i_{2} \cdots i_{d}$ deleting $k j$ 's. Then

$$
(k+1)\left|c_{i_{2} \cdots i_{d}}^{j}\right|=(k+1) C_{d}^{k+1} \beta_{j, k}=d C_{d-1}^{k} \beta_{j, k}=d\left|c_{i_{2} \cdots i_{d}}\right| .
$$

Substituting in (3.7) with the above equality, we have

$$
\begin{aligned}
\frac{\partial\left(\mathcal{A} x^{d}\right)_{i}}{\partial x_{j}} & =\sum_{k=0}^{d-1} \sum_{i_{2} \cdots i_{d} \in \Omega_{2, d}^{j, k}} d\left|c_{i_{2} \cdots i_{d}}\right| a_{i j i_{2} \cdots i_{d}} x_{i_{2}} \cdots x_{i_{d}} \\
& =d\left(\mathcal{A} x^{d-1}\right)_{i j},
\end{aligned}
$$

for $i, j=1, \cdots, n$. Hence we obtain (3.5) by the simple computation of other elements in $F^{\prime}(x, \lambda)$. 
It is noted that the expression of $\frac{\partial\left(\mathcal{A} x^{d}\right)_{i}}{\partial x_{j}}$ will be complex if $\mathcal{A}$ is not semi-symmetric. Hence the property of semi-symmetric benefits the computation of Jacobian matrix. In the following, we give Newton method to find $\lambda^{*}>0$ and $x^{*}>0$ such that $F\left(x^{*}, \lambda^{*}\right)=0$.

\section{Algorithm 3.1 Newton Method}

Step 0. Choose initial values. Choose $x_{1} \in \Re_{+}^{n}, \lambda_{0}>\sigma>0$, let $k=1$.

Step 1. Determine $\left(\Delta x_{k}, \Delta \lambda_{k}\right)$ by solving

$$
F\left(x_{k}, \lambda_{k}\right)+F^{\prime}\left(x_{k}, \lambda_{k}\right)\left(\begin{array}{c}
\Delta x_{k} \\
\Delta \lambda_{k}
\end{array}\right)=0,
$$

where $F\left(x_{k}, \lambda_{k}\right)$ and $F^{\prime}\left(x_{k}, \lambda_{k}\right)$ are defined by (3.3) and (3.5).

Step 2. Compute new iterate point,

$$
\left(x_{k+1}, \lambda_{k+1}\right)=\left(x_{k}, \lambda_{k}\right)+\left(\Delta x_{k}, \Delta \lambda_{k}\right) .
$$

Step 3. Check termination condition. If $\left\|F\left(x_{k+1}, \lambda_{k+1}\right)\right\|_{2}=0$, then stop and set $\left(x^{*}, \lambda^{*}\right)=\left(x_{k+1}, \lambda_{k+1}\right) ;$ otherwise $k=k+1$, go to Step 1 .

\section{Convergence of algorithm}

Now we discuss convergence of Algorithm 3.1. It is easy to see $F(x, \lambda)$ is continuously differentiable in $R_{++}^{n+1}$. From Lemma 3.2 we know that there exists an $\left(x^{*}, \lambda^{*}\right)$ in $R_{+}^{n+1}$ such that $F\left(x^{*}, \lambda^{*}\right)=0$. The following conclusion is important for the feasibility and convergence of Algorithm 3.1.

Lemma 4.1 Assume that $P$ is nonnegative and its associated semi-symmetric tensor $\mathcal{A}_{s}$ is irreducible, $F(x, \lambda)$ is defined by $(3.3),\left(x^{*}, \lambda^{*}\right) \in R_{++}^{n+1}$ such that $F\left(x^{*}, \lambda^{*}\right)=0$. Then $F^{\prime}\left(x^{*}, \lambda^{*}\right)$ is nonsingular.

Proof Let $P(x)=\mathcal{A}_{s} x^{d}$. Then from Lemma 2.1, $\mathcal{A}_{s}$ is nonnegative.

Let $\left(z^{T}, t\right)^{T} \in R^{n+1}$ and

$$
0=F^{\prime}\left(x^{*}, \lambda^{*}\right)\left(\begin{array}{l}
z \\
t
\end{array}\right)=\left(\begin{array}{c}
d\left(\left[\left(\sigma \mathcal{U}+\mathcal{A}_{s}\right) x^{*(d-1)}\right]^{T}-\lambda^{*} \operatorname{diag}\left(x^{*[d-1]}\right)\right) z-t x^{*[d]} \\
-\left(x^{*[d]}\right)^{T} z
\end{array}\right),
$$

where the second equality is determined by (3.5).

Multiplying the first equation by $x^{*}$ in left-side, we have

$$
d\left(\left(\sigma \mathcal{U}+\mathcal{A}_{s}\right) x^{* d}-\lambda^{*} x^{*[d]}\right)^{T} z-t \sum_{i=1}^{n}\left(x_{i}^{*}\right)^{d+1}=0 .
$$

Because $F\left(x^{*}, \lambda^{*}\right)=0$, i.e. $\left(\sigma \mathcal{U}+\mathcal{A}_{s}\right) x^{* d}=\lambda^{*} x^{*[d]}$ and $x^{*}>0$, we have $t=0$. Substituting into the first equation of (4.1), we have

$$
\left(\left(\sigma \mathcal{U}+\mathcal{A}_{s}\right) x^{*(d-1)}-\lambda^{*} \operatorname{diag}\left(x^{*[d-1]}\right)\right) z=0 .
$$

Let $Q=\left(\sigma \mathcal{U}+\mathcal{A}_{s}\right) x^{*(d-1)}-\lambda^{*} \operatorname{diag}\left(x^{*[d-1]}\right)$. Before proving $z=0$, we prove that $Q$ is irreducible. If $Q$ is not irreducible, then there exists a nonempty proper index subset $I \subset\{1,2, \cdots, n\}$ such that

$$
q_{i j}=0, \forall i \in I, \forall j \notin I \text {. }
$$


The off-diagonal elements of $Q$ are those of $\mathcal{A}_{s} x^{*(d-1)}$, i.e.

$$
q_{i j}=\sum_{i_{2} \cdots i_{d}}^{n} a_{i j i_{2} \cdots i_{d}} x_{i_{2}}^{*} \cdots x_{i_{d}}^{*}, \quad \forall i \neq j .
$$

By $\mathcal{A}_{s} \geq 0$ and $x^{*}>0,(4.3)$ means that

$$
a_{i j i_{2} \cdots i_{d}}=0, \forall i \in I, \forall j \notin I, i_{2}, \cdots, i_{d}=1, \cdots, n,
$$

which contradicts the fact that $\mathcal{A}_{s}$ is irreducible (see (1.4)).

The diagonal elements of $Q$ may be not positive, however there exists a positive number $\delta>0$ such that $(\delta E+Q) \geq 0$, where $E$ is unit matrix. Meanwhile, $\delta E+Q$ is irreducible, and by (4.2)

$$
(\delta E+Q) z=\delta z .
$$

If $z \neq 0$, then according to Perron-Frobenius theorem of a nonnegative irreducible matrix, $z>0$ or $z<0$. However the second equation of (4.1) means $z^{T} x^{*[d]}=0$, thus $z=0$.

Hence $(z, t)=0$ which implies that $F^{\prime}\left(x^{*}, \lambda^{*}\right)$ is nonsingular.

Lemma 4.2 Assume $\left(x^{*}, \lambda^{*}\right) \in R_{++}^{n+1}$ such that $F\left(x^{*}, \lambda^{*}\right)=0$. Then there exists a constant $c_{1}>0$ such that

$$
\left\|F^{\prime}(x, \lambda)-F^{\prime}\left(x^{*}, \lambda^{*}\right)\right\| \leq c_{1}\left\|(x, \lambda)-\left(x^{*}, \lambda^{*}\right)\right\|, \forall(x, \lambda) \in D
$$

where $D$ is the neighborhood of $\left(x^{*}, \lambda^{*}\right)$.

Proof From the definition, we have

$$
\begin{aligned}
& F^{\prime}(x, \lambda)-F^{\prime}\left(x^{*}, \lambda^{*}\right) \\
= & \left(\begin{array}{cc}
d\left\{\left(A x^{(d-1)}-A x^{*(d-1)}\right)-\left(\lambda \operatorname{diag}\left(x^{[d-1]}\right)-\lambda^{*} \operatorname{diag}\left(x^{*[d-1]}\right)\right)\right\} & x^{*[d]}-x^{[d]} \\
\left(x^{*[d]}-x^{[d]}\right)^{T} & 0
\end{array}\right) .
\end{aligned}
$$

By direct computation, we obtain that

$$
\begin{aligned}
& A x^{(d-1)}-A x^{*(d-1)} \\
= & A x^{(d-1)}-A x^{(d-2)} x^{*}+A x^{(d-2)} x^{*}-\cdots+A x x^{*(d-2)}-A x^{*(d-1)} \\
= & {\left[A x^{(d-2)}+A x^{(d-3)} x^{*}+\cdots+A x^{*(d-2)}\right]\left(x-x^{*}\right), } \\
& x^{*[d]}-x^{[d]} \\
= & x^{*[d]}-x^{*[d-1]} \circ x+x^{*[d-1]} \circ x-\cdots+x^{[d-1]} \circ x^{*}-x^{[d]} \\
= & \operatorname{diag}\left(x^{*[d-1]}+x^{*[d-2]} \circ x+\cdots+x^{[d-1]}\right)\left(x^{*}-x\right),
\end{aligned}
$$

and

$$
\begin{aligned}
& \lambda \operatorname{diag}\left(x^{[d-1]}\right)-\lambda^{*} \operatorname{diag}\left(x^{*[d-1]}\right) \\
= & \lambda \operatorname{diag}\left(x^{[d-1]}\right)-\lambda^{*} \operatorname{diag}\left(x^{[d-1]}\right)+\lambda^{*} \operatorname{diag}\left(x^{[d-1]}\right)-\lambda^{*} \operatorname{diag}\left(x^{*[d-1]}\right) \\
= & \left(\lambda-\lambda^{*}\right) \operatorname{diag}\left(x^{[d-1]}\right)+\lambda^{*} \operatorname{diag}\left(x^{[d-1]}-x^{*[d-1]}\right), \\
= & \left(\lambda-\lambda^{*}\right) \operatorname{diag}\left(x^{[d-1]}\right)+\lambda^{*} \operatorname{diag}\left(x^{[d-2]}+x^{[d-3]} \circ x^{*}+\cdots+x^{*[d-2]}\right) \operatorname{diag}\left(x-x^{*}\right) .
\end{aligned}
$$

Substituting into (4.4) with (4.5)-(4.7), we obtain the conclusion of this lemma from basic property of matrix and vector norm. 
Denote $w_{k}=\left(x_{k}, \lambda_{k}\right), p_{k}=\left(\Delta x_{k}, \Delta \lambda_{k}\right)$. The quadratic convergence of Algorithm 3.1 is given below.

Theorem 4.1 Let $P$ and $\mathcal{A}$ be a nonnegative homogeneous map of degree $d$ and its associated semi-symmetric tensor, respectively. Assume that $\mathcal{A}$ is irreducible. Then we have the following conclusions.

(1) There is an open set $S$ which contains $w^{*}=\left(x^{*}, \lambda^{*}\right)$ such that for any $w_{0} \in S$ the iterates $w_{k}$ generated by Algorithm 3.1 are well-defined, remain in $S$ and converge to $w^{*}$.

(2) There is a constant $\beta>0$ such that

$$
\left\|w_{k+1}-w^{*}\right\| \leq \beta\left\|w_{k}-w^{*}\right\|^{2}, k=0,1,2, \cdots .
$$

Proof The conclusion in this theorem is obtained by Lemmas 4.1 and 4.2, Theorem 2.1 in [4].

In order to obtain a globally convergent algorithm, we use the global technique in Algorithm 3.1. A merit function $f(x)=\frac{1}{2}\|F(x)\|^{2}$ is chosen, $p_{k}$ is modified such that

$$
-p_{k}^{T} \nabla f\left(w_{k}\right) \geq \delta\left\|p_{k}\right\|\left\|\nabla f\left(w_{k}\right)\right\|,
$$

a line search is used to find a steplength $t_{k} \in(0,1]$ such that

$$
\begin{gathered}
f\left(w_{k}+t_{k} p_{k}\right) \leq f\left(w_{k}\right)+\rho t_{k} \nabla f\left(w_{k}\right)^{T} p_{k}, \\
\nabla f\left(w_{k}+t_{k} p_{k}\right)^{T} p_{k} \geq \bar{\sigma} \nabla f\left(w_{k}\right)^{T} p_{k},
\end{gathered}
$$

and

$$
w_{k}+t_{k} p_{k} \in \Re_{++}^{n+1}
$$

where $\rho \in(0,0.5), \bar{\sigma} \in(\rho, 1), \delta \in(0,1)$.

\section{Algorithm 4.1 Global Newton method}

Step 0. Choose initial values. Choose $\rho \in(0,0.5), \bar{\sigma} \in(\rho, 1), \delta \in(0,1), \epsilon \geq 0, w_{1} \in$ $\Re_{++}^{n+1}$, where $w_{n+1}^{(1)}>\sigma>0, k=1$.

Step 1. Find $p_{k}=\left(\Delta x_{k}, \Delta \lambda_{k}\right)$ by solving (3.8). If (4.8) is not satisfied, then choose $\tau_{k}$ and compute

$$
p_{k}=-\left(\left(F_{k}^{\prime}\right)^{T} F_{k}^{\prime}+\tau_{k} I\right)^{-1} \nabla f\left(w_{k}\right),
$$

such that (4.8) is satisfied.

Step 2. Find a steplength $t_{k} \in(0,1]$ satisfying (4.9),(4.10) and (4.11). Set

$$
w_{k+1}=w_{k}+t_{k} p_{k} .
$$

Step 3 Check termination condition. If $f\left(w_{k+1}\right) \leq \epsilon$, then $\operatorname{set}\left(x^{*}, \lambda^{*}\right)=w_{k+1}$ and stop; otherwise $k=k+1$, go to Step 1 .

Remark In Step 1, if (4.8) is not satisfied, then the technique in [12, pages 264-265] is used to choose $\tau_{k}$. According to Lemma 3.1 in [12], it is not difficult to find a $t_{k}$ which can satisfy (4.9),(4.10) and (4.11).

In order to discuss the global convergence, we define the level set

$$
L\left(w_{0}\right)=\left\{w \mid f(w) \leq f\left(w_{0}\right)\right\}
$$

for $w_{0} \in R^{n+1}$. 
Theorem 4.2 Suppose that $F^{\prime}(w)$ is Lipschitz continuous in $L\left(w_{0}\right)$. Then we have

$$
\lim _{k \rightarrow \infty} \nabla f\left(w_{k}\right)=0
$$

Proof This theorem follows Theorem 3.2 in [12] and the condition (4.8).

Theorem 4.3 Let $P$ be nonnegative and its associated semi-symmetric tensor $\mathcal{A}_{s}$ be irreducible, $F: \Re_{++}^{n+1} \rightarrow \Re^{n+1}$ be defined by (3.3), $w_{0} \in \Re_{++}^{n+1}$. Assume that $F^{\prime}(w)$ is Lipschitz continuous in $L\left(w_{0}\right), t_{k}=1$ is admissible and (4.8) is satisfied for all $k$ greater than a certain index $k_{0}, F^{\prime}\left(w^{*}\right)$ is nonsingular where $w^{*}$ is a limited point of $\left\{w_{k}\right\}$ generated by Algorithm 4.1. Then $\left\{w_{k}\right\}$ quadratically converges to $w^{*}$.

Proof By Theorem 4.2, we have

$$
\lim _{k \rightarrow \infty} \nabla f\left(w_{k}\right)=0
$$

where $\nabla f\left(w_{k}\right)=F^{\prime}\left(w_{k}\right) F\left(w_{k}\right)$. Hence, $\left\{w_{k}\right\}$ is convergent. Let $w^{*}$ be the limited point. Because $F^{\prime}\left(w^{*}\right)$ is nonsingular, we obtain

$$
\lim _{k \rightarrow \infty} F\left(w_{k}\right)=0 .
$$

From the assumption, we know that there exists $k_{0}$ such that $t_{k}=1$ is admissible and (4.8) is satisfied for all $k \geq k_{0}$. This means that $w_{k}$ can be generated by Algorithm 3.1 for $k>k_{0}$. The conclusion is obtained by Theorem 4.1 .

\section{Numerical test}

In this section, we present some preliminary numerical tests. We use Algorithm 4.1 to compute some randomly generated problems. The computation was done on a personal computer (Pentium IV, 1.83GHz,512MB) running Matlab 7.0.

Because it is difficult to find test problems in the literature, we generate some problems by the random approaches for testing the performance of Algorithm 4.1. In the following problems, let $P(x)=\mathcal{A} x^{d} \in \Re^{n}$ where $d$ is odd.

TP I and TP II are generated from some similar problems in [8].

TP I (special case) $P(x)=A x^{d}$ is defined as

$$
p(x)=\left(\begin{array}{c}
\left(a_{1}+\gamma\right) x_{1}^{d}+0.5\left(\sum_{i=1}^{n} b_{i} x_{i} \sum_{i=1}^{n} c_{i} x_{i}\right)^{\frac{d-1}{2}}\left(c_{1} \sum_{i=1}^{n} b_{i} x_{i}+b_{1} \sum_{i=1}^{n} c_{i} x_{i}\right) \\
\ldots \\
\left(a_{j}+\gamma\right) x_{j}^{d}+0.5\left(\sum_{i=1}^{n} b_{i} x_{i} \sum_{i=1}^{n} c_{i} x_{i}\right)^{\frac{d-1}{2}}\left(c_{j} \sum_{i=1}^{n} b_{i} x_{i}+b_{j} \sum_{i=1}^{n} c_{i} x_{i}\right) \\
\ldots \\
\ldots \\
\left(a_{n}+\gamma\right) x_{n}^{d}+0.5\left(\sum_{i=1}^{n} b_{i} x_{i} \sum_{i=1}^{n} c_{i} x_{i}\right)^{\frac{d-1}{2}}\left(c_{n} \sum_{i=1}^{n} b_{i} x_{i}+b_{n} \sum_{i=1}^{n} c_{i} x_{i}\right)
\end{array}\right)
$$

where $a_{i}, b_{i}, c_{i}(i=1, \cdots, n)$ are random number in $[0,1], \gamma>0$ is a parameter. 
Table 1 Results of Problem I

\begin{tabular}{|c|c|c|c|c|c|c|c|c|}
\hline \multicolumn{3}{|c|}{ TPI } & \multicolumn{3}{|c|}{ Algorithm 4.1} & \multicolumn{3}{|c|}{ Power algorithm } \\
\hline $\bar{d}$ & $n$ & $\gamma$ & iter & term & $\mathrm{cpu}$ & iter & term & $\mathrm{cpu}$ \\
\hline 3 & 20 & 10 & 8 & $8.1564 \mathrm{e}-6$ & 0.0110 & 7 & $4.3884 \mathrm{e}-7$ & 0.0050 \\
\hline 3 & 20 & $10^{2}$ & 9 & $1.1396 \mathrm{e}-13$ & 0.0094 & 14 & $4.2976 \mathrm{e}-6$ & 0.0060 \\
\hline 3 & 20 & $10^{3}$ & 8 & $6.8536 \mathrm{e}-6$ & 0.0069 & 57 & $9.7904 \mathrm{e}-6$ & 0.0113 \\
\hline 3 & 20 & $10^{4}$ & 9 & 0.00 & 0.0060 & 171 & $9.5596 \mathrm{e}-6$ & 0.0335 \\
\hline 3 & 60 & $10^{2}$ & 10 & $3.6380 \mathrm{e}-12$ & 0.0329 & 9 & $1.0862 \mathrm{e}-6$ & 0.0160 \\
\hline 3 & 60 & $10^{3}$ & 10 & $7.2760 \mathrm{e}-12$ & 0.0172 & 10 & $3.9026 \mathrm{e}-6$ & 0.0063 \\
\hline 3 & 60 & $10^{4}$ & 10 & $3.6380 \mathrm{e}-12$ & 0.0132 & 22 & $4.1141 \mathrm{e}-6$ & 0.0115 \\
\hline 3 & 60 & $10^{5}$ & 10 & $3.7774 \mathrm{e}-10$ & 0.0123 & 160 & $9.7867 \mathrm{e}-6$ & 0.0740 \\
\hline 3 & 100 & $10^{3}$ & 10 & $2.1849 \mathrm{e}-7$ & 0.0198 & 9 & $7.8849 \mathrm{e}-7$ & 0.0088 \\
\hline 3 & 100 & $10^{4}$ & 10 & $2.2774 \mathrm{e}-7$ & 0.0186 & 13 & $2.9888 \mathrm{e}-6$ & 0.0110 \\
\hline 3 & 100 & $10^{5}$ & 10 & $2.8368 \mathrm{e}-7$ & 0.0184 & 43 & $6.4058 \mathrm{e}-6$ & 0.0335 \\
\hline 3 & 100 & $10^{6}$ & 10 & $2.4005 \mathrm{e}-7$ & 0.0192 & 291 & $9.7326 \mathrm{e}-6$ & 0.2133 \\
\hline
\end{tabular}

TP II (special case) $P(x)=A x^{d}$ is defined as

$$
p(x)=\left(\begin{array}{c}
\left(a_{1}+\gamma\right) x_{1}^{d}+0.5\left(\sum_{i=1}^{n} b_{i} x_{i}\right)^{\frac{d+1}{2}} x_{1}^{\frac{d-1}{2}}+0.5\left(\sum_{i=1}^{n} x_{i}^{\frac{d+1}{2}}\right)\left(\sum_{i=1}^{n} b_{i} x_{i}\right)^{\frac{d-1}{2}} b_{1} \\
\cdots \\
\left(a_{j}+\gamma\right) x_{j}^{d}+0.5\left(\sum_{i=1}^{n} b_{i} x_{i}\right)^{\frac{d+1}{2}} x_{j}^{\frac{d-1}{2}}+0.5\left(\sum_{i=1}^{n} x_{i}^{\frac{d+1}{2}}\right)\left(\sum_{i=1}^{n} b_{i} x_{i}\right)^{\frac{d-1}{2}} b_{j} \\
\ldots \\
\left(a_{n}+\gamma\right) x_{n}^{d}+0.5\left(\sum_{i=1}^{n} b_{i} x_{i}\right)^{\frac{d+1}{2}} x_{n}^{\frac{d-1}{2}}+0.5\left(\sum_{i=1}^{n} x_{i}^{\frac{d+1}{2}}\right)\left(\sum_{i=1}^{n} b_{i} x_{i}\right)^{\frac{d-1}{2}} b_{n}
\end{array}\right)
$$

where $a_{i}, b_{i}(i=1, \cdots, n)$ are random number in $[0,1], \gamma>0$ is a parameter.

TP III (general case)

$$
p(x)=\operatorname{Mr}(x)+\gamma x^{d},
$$

where $p(x)$ is homogeneous polynomial map with the degree $d, M=\left(m_{i j}\right) \in \Re^{n \times u_{d}}, u_{d}$ is defined in (2.5), $m_{i j}\left(i=1, \cdots, n ; j=1, \cdots, u_{d}\right)$ are random numbers in $[0,1], \gamma>0$ is a parameter.

Algorithm 4.1 and the power algorithm in [5] are used to solve these problems. Tables 1, 2 and 3 list the numerical results of TP I-III where $d, n$ are the number of dimension and order in tensor, $\gamma$ is chosen from 10 to $10^{7}$, iter is the number of iterations, cpu is cpu time in seconds. In order to compare these two methods, we choose the same initial point $x_{0}=(1, \cdots, 1)^{T}$, and the same termination conditions

$$
\left\|\lambda_{k}-\lambda_{k+1}\right\| \leq 10^{-5},\left\|P\left(x_{k+1}\right)-\lambda_{k+1} x_{k+1}^{[d]}\right\|_{2} \leq 10^{-5} .
$$

If the termination conditions are not satisfied in 500 iteration, then the algorithm stops. In addition, term is the last value of $\left\|\lambda_{k}-\lambda_{k+1}\right\|$.

The results reported in Tables 1, 2 and 3 show that Algorithm 4.1 performs well for these test problems. Although $\gamma$ changes a lot, there is only subtle changes in the number of iterations of Algorithm 4.1. When $\gamma$ is relative small, the number of iteration of the Power 
Table 2 Results of Problem II

\begin{tabular}{|c|c|c|c|c|c|c|c|c|}
\hline \multicolumn{3}{|c|}{ TPII } & \multicolumn{3}{|c|}{ Algorithm 4.1} & \multicolumn{3}{|c|}{ Power algorithm } \\
\hline $\bar{d}$ & $n$ & $\gamma$ & iter & term & $\mathrm{cpu}$ & iter & term & $\mathrm{cpu}$ \\
\hline 3 & 20 & 10 & 9 & $3.5135 \mathrm{e}-8$ & 0.0081 & 14 & $9.2605 \mathrm{e}-6$ & 0.0065 \\
\hline 3 & 20 & $10^{2}$ & 9 & $6.5457 \mathrm{e}-8$ & 0.0075 & 30 & $9.6946 \mathrm{e}-6$ & 0.0088 \\
\hline 3 & 20 & $10^{3}$ & 9 & $5.9262 \mathrm{e}-8$ & 0.0062 & 211 & $9.8138 \mathrm{e}-6$ & 0.0359 \\
\hline 3 & 20 & $10^{4}$ & 9 & $3.0865 \mathrm{e}-8$ & 0.0065 & $>500$ & & \\
\hline 3 & 60 & 10 & 9 & $4.5475 \mathrm{e}-13$ & 0.0257 & 15 & $9.6892 \mathrm{e}-6$ & 0.0097 \\
\hline 3 & 60 & $10^{2}$ & 9 & $6.8212 \mathrm{e}-13$ & 0.0130 & 17 & $5.0157 \mathrm{e}-6$ & 0.0112 \\
\hline 3 & 60 & $10^{3}$ & 9 & $6.4213 e-13$ & 0.0112 & 38 & $7.6581 \mathrm{e}-6$ & 0.0111 \\
\hline 3 & 60 & $10^{4}$ & 9 & $1.8190 \mathrm{e}-12$ & 0.0110 & 178 & $9.3875 \mathrm{e}-6$ & 0.0430 \\
\hline 3 & 100 & 10 & 9 & $1.6151 \mathrm{e}-8$ & 0.0288 & 16 & $6.6040 \mathrm{e}-6$ & 0.0212 \\
\hline 3 & 100 & $10^{2}$ & 9 & $1.1550 \mathrm{e}-8$ & 0.0142 & 16 & $7.1325 \mathrm{e}-6$ & 0.0105 \\
\hline 3 & 100 & $10^{3}$ & 9 & $1.5506 \mathrm{e}-8$ & 0.0147 & 25 & $6.5653 e-6$ & 0.0103 \\
\hline 3 & 100 & $10^{4}$ & 9 & $1.4251 \mathrm{e}-8$ & 0.0148 & 84 & $9.9054 \mathrm{e}-6$ & 0.0333 \\
\hline 7 & 100 & $10^{4}$ & 10 & $9.6392 \mathrm{e}-7$ & 0.0226 & 25 & $9.9586 \mathrm{e}-6$ & 0.0111 \\
\hline 7 & 100 & $10^{5}$ & 11 & $9.3132 \mathrm{e}-10$ & 0.0467 & 29 & $6.2184 \mathrm{e}-6$ & 0.0223 \\
\hline 7 & 100 & $10^{6}$ & 11 & $1.8626 \mathrm{e}-9$ & 0.0277 & 34 & $6.0468 \mathrm{e}-6$ & 0.0166 \\
\hline 7 & 100 & $10^{7}$ & 11 & $3.7253 \mathrm{e}-9$ & 0.0200 & 87 & $7.8995 \mathrm{e}-6$ & 0.0355 \\
\hline
\end{tabular}

Table 3 Results of Problem III

\begin{tabular}{|c|c|c|c|c|c|c|c|c|}
\hline \multicolumn{3}{|c|}{ TPIII } & \multicolumn{3}{|c|}{ Algorithm 4.1} & \multicolumn{3}{|c|}{ Power algorithm } \\
\hline $\bar{d}$ & $n$ & $\gamma$ & iter & term & $\mathrm{cpu}$ & iter & term & $\mathrm{cpu}$ \\
\hline 3 & 20 & $10^{2}$ & 8 & $4.5475 \mathrm{e}-13$ & 0.1833 & 9 & $3.7559 \mathrm{e}-6$ & 0.0806 \\
\hline 3 & 20 & $10^{3}$ & 8 & $1.3188 \mathrm{e}-11$ & 0.1864 & 27 & $6.9269 \mathrm{e}-6$ & 0.2029 \\
\hline 3 & 20 & $10^{4}$ & 8 & $2.3481 \mathrm{e}-7$ & 0.1919 & 164 & $9.5877 \mathrm{e}-6$ & 1.2369 \\
\hline 3 & 20 & $10^{5}$ & 9 & $1.3097 \mathrm{e}-10$ & 0.2127 & $>500$ & & \\
\hline 3 & 40 & $10^{2}$ & 8 & $9.2580 \mathrm{e}-8$ & 2.2022 & 6 & $5.0420 \mathrm{e}-6$ & 0.8786 \\
\hline 3 & 40 & $10^{3}$ & 8 & $8.8839 \mathrm{e}-7$ & 2.1601 & 11 & $2.5065 \mathrm{e}-6$ & 1.5659 \\
\hline 3 & 40 & $10^{4}$ & 8 & $8.9635 \mathrm{e}-6$ & 2.1639 & 35 & $8.0440 \mathrm{e}-6$ & 4.8113 \\
\hline 3 & 40 & $10^{5}$ & 9 & $4.8021 \mathrm{e}-10$ & 2.4448 & 225 & $9.6221 \mathrm{e}-6$ & 32.228 \\
\hline 3 & 60 & $10^{2}$ & 9 & $1.4522 \mathrm{e}-11$ & 17.6957 & 6 & $1.7072 \mathrm{e}-7$ & 8.3350 \\
\hline 3 & 60 & $10^{3}$ & 9 & $1.0914 \mathrm{e}-11$ & 17.7530 & 8 & $1.8095 \mathrm{e}-6$ & 11.4625 \\
\hline 3 & 60 & $10^{4}$ & 9 & $6.8556 \mathrm{e}-15$ & 17.6028 & 18 & $3.6666 \mathrm{e}-06$ & 25.2876 \\
\hline 3 & 60 & $10^{5}$ & 9 & $1.1059 \mathrm{e}-9$ & 17.7278 & 87 & $8.6087 e-6$ & 123.5715 \\
\hline
\end{tabular}

algorithm is the same as or a little more than that of Algorithm 4.1, and uses less time. While $\gamma$ increases, the Power algorithm needs more than two times of number of iteration for Algorithm 4.1, and uses more time.

From Corollary 5.2 in [5], it follows that the Power algorithm slowly converges, when $\frac{\lambda}{r}$ approaches to 1, where $r$ is the maximal modulus of the eigenvalues of $p(x)$ distinct from $\lambda$. From TPI-III, it is easy to see that as $\gamma$ is larger and larger, $\frac{\lambda}{r}$ is very close to 1 . Hence, it is not strange that the Power algorithm needs more time and more number of iterations to solve TPI-III when $\gamma$ is large.

Table 4 lists the numerical result of two algorithms which solves TPIII when $d=3, n=$ $40, \gamma=10,000$, which show the quadratic convergence of Algorithm 4.1 and linear convergence of the Power algorithm. 
Table 4 Convergent rate

\begin{tabular}{lll}
\hline $\mathrm{k}$ & $\begin{array}{l}\text { Algorithm } 4.1 \\
\text { term }\end{array}$ & $\begin{array}{l}\text { Power algorithm } \\
\text { term }\end{array}$ \\
\hline 1 & $2.6880 \mathrm{e}+3$ & $1.5818 \mathrm{e}+4$ \\
2 & $2.2477 \mathrm{e}+3$ & $2.6090 \mathrm{e}+1$ \\
3 & $2.0965 \mathrm{e}+3$ & $1.6462 \mathrm{e}+1$ \\
4 & $2.0965 \mathrm{e}+3$ & $1.0412 \mathrm{e}+1$ \\
5 & $9.6079 \mathrm{e}+2$ & $6.5965 \mathrm{e}+0$ \\
6 & $1.0508 \mathrm{e}+2$ & $4.1832 \mathrm{e}+0$ \\
7 & $5.8060 \mathrm{e}-1$ & $2.5645 \mathrm{e}+0$ \\
8 & $8.9635 \mathrm{e}-6$ & $1.6851 \mathrm{e}+0$ \\
9 & & $1.0700 \mathrm{e}+0$ \\
10 & & $6.7950 \mathrm{e}-1$ \\
15 & & $7.0301 \mathrm{e}-2$ \\
20 & & $7.3150 \mathrm{e}-3$ \\
25 & & $4.7758 \mathrm{e}-4$ \\
30 & & $4.9415 \mathrm{e}-5$ \\
35 & & $8.0440 \mathrm{e}-6$
\end{tabular}

\section{Comments}

In this paper we propose a quadratically convergent algorithm for computing the largest eigenvalue of a nonnegative homogeneous polynomial map where the Newton method is used to solve an equivalent system of nonlinear equations. The semi-symmetric tensor is introduced to reveal the relation between homogeneous polynomial map and its associated semi-symmetric tensor. The technique of computation may be used to find the other eigenvalues of a nonnegative homogeneous polynomial map or a nonnegative tensor.

\section{References}

1. Buló, S.R., Pelillo, M.: New bounds on the clique number of graphs based on spectral hypergraph theory. In: Stützle, T. (ed.) Learning and Intelligent Optimization, pp. 45-48. Springer, Berlin (2009)

2. Chang, K.C., Pearson, K., Zhang, T.: Perron-Frobenius theorem for nonnegative tensors. Commun. Math. Sci. 6, 507-520 (2008)

3. Chang, K.C., Pearson, K., Zhang, T.: Primitivity, the convergence of the NQZ method, and the largest eigenvalue for nonnegative tensors. SIAM J. Matrix Anal. Appl. 32, 806-819 (2011)

4. Dennis Jr, J.E., More, J.J.: Quasi-Newton methods, motivation and theory. SIAM Rev. 19, 46-89 (1977)

5. Friedland, S., Gauber, S., Han, L.: Perron-Frobenius theorem for nonnegative multilinear forms and extensions. Linear Algebra Appl. 438, 738-749 (2013)

6. Hu, S., Qi, L.: Algebraic connectivity of an even uniform hypergraph. J. Comb. Optim. 24, 564-579 (2012)

7. Lim, L.H.: Singular values and eigenvalues of tensors, a variational approach. In: Proceedings of the 1st IEEE International Workshop on Computational Advances of Multi-tensor Adaptive Processing, pp. 129-132(2005)

8. Liu, Y., Zhou, G., Ibrahim, N.F.: An always convergent algorithm for the largest eigenvalue of an irreducible nonnegative tensor. J. Comput. Appl. Math. 235, 286-292 (2010)

9. Lyusternik, L., Shnirel'man L.: Topological methods in variational prob lems and their application to the differential geometry of surfaces. (Russian) Uspehi Matem. Nauk (N.S.) 2, 1(17), 166-217 (1947)

10. Ng, M., Qi, L., Zhou, G.: Finding the largest eigenvalue of a nonnegative tensor. SIAM. J. Matrix Anal. Appl. 31, 1090-1099 (2009)

11. Ni, Q., Qi, L., Wang, F.: An eigenvalue method for testing the positive definiteness of a multivariate form. IEEE Trans. Automat. Control 53, 1096-1107 (2008) 
12. Nocedal, J., Wright, S.J.: Numerical Optimization. Springer, New York (1999)

13. Qi, L.: Eigenvalues of a real supersymmetric tensor. J. Symb. Comput. 40, 1302-1324 (2005)

14. Qi, L., Teo, K.L.: Multivariate polynomial minimization and its application in signal processing. J. Global Optim. 26, 419-433 (2003)

15. Qi, L., Wang, Y., Wu, E.X.: D-eigenvalues of diffusion kurtosis tensor. J. Comput. Appl. Math. 221, 150-157 (2008)

16. Qi, L., Yu, G., Wu, E.X.: Higher order positive semi-definite diffusion tensor imaging. SIAM J. Imaging Sci. 3, 416-433 (2010)

17. Yang, Y., Yang, Q.: Further results for Perron-Frobenius Theorem for nonnegative tensors. SIAM J. Matrix Anal. Appl. 31, 2517-2530 (2010)

18. Zhang, L., Qi, L.: Linear convergence of an algorithm for computing the largest eigenvalue of a nonnegative tensor. Numer. Linear Algebra Appl. 19, 830-841 (2012)

19. Zhang, L., Qi, L., Xu, Y.: Linear convergence of the LZI algorithm for weakly positive tensors. J. Comput. Math. 30, 24-33 (2012) 